\title{
A MULTI-OPTIONAL HYBRID FUNCTIONS ENTROPY AS A TOOL FOR TRANSPORTATION MEANS REPAIR OPTIMAL PERIODICITY DETERMINATION
}

\author{
Andriy GONCHARENKO* \\ National Aviation Universit, Kosmonavta Komarova ave. 1, 03680 Kyiv, Ukraine
}

Received 27 May 2018; accepted 18 June 2018

\begin{abstract}
The paper considers theoretical explanation and construction of some mathematical models of a transportation mean operational process in reference to maintenance optimal periodicity. The important finding is that the objectively existing engineering transportation means maintenance optimal periodicity is determined in the different from the probabilistic methods way. There is a scientifically proven explanation for the mentioned above periodicity optimization with the help of the specially introduced hybrid-optional effectiveness functions distribution. The developed doctrine uses the entropy paradigm conditional extremization approach. This contribution allows obtaining the wanted optimal periodicities sidestepping the related states probabilities determination and their further extremization. The essential breakthrough of the developed doctrine is that the optional objective effectiveness functions, in such a case, are the corresponding combinations of the intensities of the studied system's possible transitions from state to state, which relates with the set of the considered operational options. Corresponding limit solutions for the zero-to-zero ratio indeterminate forms are analyzed. Theoretical speculations are illustrated with the example calculation experiments. The necessary diagrams are plotted.
\end{abstract}

Keywords: transportation mean, maintenance, optimal periodicity, hybrid-optional function, entropy, probability, extremization, multi-optional situation.

\section{Introduction}

Operation of any transportation mean requires periodical maintenance and repair. As it has been considered in references Dhillon (2006), Nakagawa (2005), and Smith (2005), issues of reliability, risks, maintainability, and maintenance ought to be taken into account by engineers. Applicably to aviation transportation (Wild \& Kroes, 2014; Kroes, Watkins, Delp, \& Sterkenburg, 2013; Smirnov et al., 1990), safety of operation greatly depends upon the timely and duly performed repair and maintenance of aircraft itself and the most important parts and systems of the airplane.

As to the aircraft powerplants and gas-turbine engines, there are quite perfectly developed progressive technologies of the engines elements restorations (Tamarin, 2002; Pallos, 2001; Dmitriyev, Koudrin, Labunets, \& Kindrachuk, 2005). Such techniques, in conjunction with the other appropriate aircraft airworthiness ensuring strategies (Thian, 2015; Le \& Lappas, 2015; Goncharenko, 2017a), provide a good quality in aviation maintenance (Gališanskis, 2004).
Regarding other types of transportation, there is a possibility to notice the similar problems of safety provisions (likewise airworthiness support in aviation). For example, in the sea-going vessels area (Klaas van Dokkum, 2005; Kuiken, 2008a, 2008b), analogous notion of seaworthiness might be deemed as an equivalent to the airworthiness in aviation, with some adequate processes of the corresponding engineering facilities technical state support (Fisher \& Hodge, 1986).

The same operational problems appear in different fields. It is worth mentioning the problems of aircraft noise assessment, prediction, and control (Zaporozhets, Tokarev, \& Attenborough, 2011); estimation of quality parameters in the radio flight support operational system (Solomentsev, Zaliskyi, \& Zuiev, 2016); synergy of piloted, remotely piloted and unmanned air systems in single air navigation space (Chepizenko, Kharchenko, \& Pavlova, 2013); consequences of shallow flows of liquid on the airport runways and automobile roads (Beljatynskij, Prentkovskis, \& Krivenko, 2010).

${ }^{*}$ Corresponding author. E-mail: andygoncharenco@yahoo.com 
In any case, the optimal periodicity for maintenance and repair carrying out is very important and it must be predetermined in order to avoid possible damages and troubles related with the probable crashes. Although forecasting of the engineering technical states is rather well developed in reliability theory, still, there is a deficiency of some new scientific approaches for explanations of probabilistic phenomena on some other, different from the traditional form, basis.

A few attempts in this direction have been made in some particular cases (Goncharenko, 2017b, 2018b, $2018 c)$. Still, there is a necessity of further theoretical developments, with possible generalizations. The presented paper contains one of such proposed approaches.

Besides, sometimes there is a lack of the theoretical considerations with regard to the mathematical limit solutions in cases with the indeterminate forms, for instance, the case of the aeronautical engineering maintenance optimal periodicity determination (Smirnov et al., 1990, SubChapter 15.4 , pp. 168-173, especially p. 171 (15.1) and on). There is the indeterminate form of the ratio of the $\frac{0}{0}$ kind for the probability of the damaged state, as well as for the optimal periodicity there.

Therefore, the presented paper is going to deal mostly with those theoretical research gaps mentioned above.

An investigation tool, which is going to be used in the presented research, is similar to subjective analysis (Kasianov, 2013; Goncharenko, 2016, 2017a). However, the developed herewith doctrine uses just objective characteristics of a system's functioning and only. For the present moment, this doctrine, applying the entropy paradigm (like the one proposed in subjective analysis) is undergoing an evolution loop. The doctrine is entering the scientific sphere operating with both:

1) the objectively existing parameters of the considered processes and

2) multi-optional hybrid functions of a special kind, which are composed for discovering some sought after optimal (in a certain respect) values with taking into account the uncertainty of the functions "multi-optionality" (Goncharenko, 2016, 2017a, 2017b, 2018a, 2018c).

\section{Schematic consideration of the problem statement}

In order to formulate the problem presented in this research about a transportation mean operation processes of maintenance and technical state support, with taking into account multi-optionality of the processes, it is recommended to consider schematically the transportation mean operation process, depending upon the options, shown in Figure 1.

In Figure 1, three states are depicted as follows: "0" is the initial state of the transportation mean, it is supposed to be in the up state (best working conditions, for example, from the manufacturing, or overhaul, complete restoration

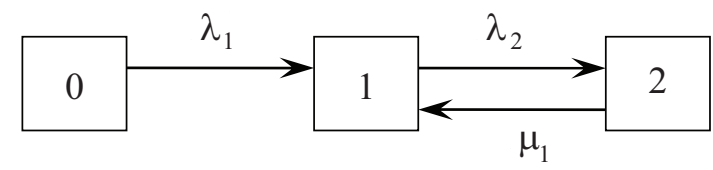

Figure 1. Graph of states of a system with a degrading and possible partial restoration

of the abilities); " 1 " is the state with the partially good (not excellent but not bad or poor) technical conditions as compared with the initial "0" state. Functional abilities of the transportation mean are not as much as that for the initial " 0 " state; the system is degrading gradually. " 2 " is the state with the worst allowable state (the transportation mean is not in a good as that described with " 1 " state but still working, functioning, running). The arrows symbolize certain transitions from state to state with the intensities denoted as $\lambda_{1}, \lambda_{2}$, and $\mu_{1}$, and the corresponding directions of the transitions.

Such problem formulation is a simplified and one of the possible, similar to the discussed at references (Goncharenko, 2017b, 2018b, 2018c). However, the presented hereafter problem is significantly different form the previous since there is no state without an exit. Nonetheless, there is a group of states with no exit.

\section{General methods of research}

The problem statement is such as for some Markovian random process; however, the options of the process, hybrid optional functions, their degree of uncertainty and the proposed optimization method (different from the traditional classical probabilistic approach, yielding the same correct result although) appears to be the intrinsic matter.

\subsection{Classical probabilistic approach}

It is assumed that the flow of random events transferring the system from state to state is the simplest one.

Then, it is possible to compose the system of ordinary differential equations (by Erlang) of the first order (such method has also been applied in references (Goncharenko, 2017b, 2018b, 2018c), although the system is different, herewith the presented paper, in accordance with the stated problem setting illustrated with the scheme represented in Figure 1), in order to determine the probabilities of the states as the dynamical characteristics of the system development in time:

$$
\left.\begin{array}{l}
\frac{d P_{0}}{d t}=-\lambda_{1} P_{0} ; \\
\frac{d P_{1}}{d t}=\lambda_{1} P_{0}-\lambda_{2} P_{1}+\mu_{1} P_{2} ; \\
\frac{d P_{2}}{d t}=\lambda_{2} P_{1}-\mu_{1} P_{2} .
\end{array}\right\}
$$

Where, $P_{0}, P_{1}$, and $P_{2}$ are probabilities of the corresponding states; $t$ - time. 
The system of the ordinary linear differential equations of (1) can be solved either with the help of Laplace transformations or in another way, for instance, applying characteristic equation:

$$
\left|\begin{array}{ccc}
-\left(\lambda_{1}\right)-k & 0 & 0 \\
\lambda_{1} & -\left(\lambda_{2}\right)-k & \mu_{1} \\
0 & \lambda_{2} & -\left(\mu_{1}\right)-k
\end{array}\right|=0
$$

where $k$ is parameter to be determined for finding the probabilities of $P_{0}, P_{1}$, and $P_{2}$ of the system corresponding states.

In case of Laplace transformations usage, similar to system (1), there is the system of transformed algebraic equations with respect to the transformation parameter $p$ and at the initial conditions of $P_{0}=1, P_{1}=0$, and $P_{2}=0$ :

$$
\left.\begin{array}{l}
p F_{0}(p)-1=-\left(\lambda_{1}\right) F_{0}(p) ; \\
p F_{1}(p)-0=\lambda_{1} F_{0}(p)-\left(\lambda_{2}\right) F_{1}(p)+\mu_{1} F_{2}(p) ; \\
p F_{2}(p)-0=\lambda_{2} F_{1}(p)-\mu_{1} F_{2}(p),
\end{array}\right\}
$$

where $F_{0}(p), F_{1}(p)$, and $F_{2}(p)$ are corresponding transformants (images) of the original (initial) probabilities of $P_{0}(t), P_{1}(t)$, and $P_{2}(t)$.

The solution for the transformants of the system of (3) yields:

$$
F_{0}(p)=\frac{p^{2}+p\left(\mu_{1}+\lambda_{2}\right)}{p\left\{p^{2}+p\left(\mu_{1}+\lambda_{2}+\lambda_{1}\right)+\left[\left(\lambda_{1} \mu_{1}\right)+\left(\lambda_{1} \lambda_{2}\right)\right]\right\}} .
$$

$$
F_{1}(p)=\frac{p \lambda_{1}+\lambda_{1} \mu_{1}}{p\left\{p^{2}+p\left(\mu_{1}+\lambda_{2}+\lambda_{1}\right)+\left[\left(\lambda_{1} \mu_{1}\right)+\left(\lambda_{1} \lambda_{2}\right)\right]\right\}} \text {. }
$$

$$
F_{2}(p)=\frac{\lambda_{1} \lambda_{2}}{p\left\{p^{2}+p\left(\mu_{1}+\lambda_{2}+\lambda_{1}\right)+\left[\left(\lambda_{1} \mu_{1}\right)+\left(\lambda_{1} \lambda_{2}\right)\right]\right\}} .
$$

Making inverse Laplace transformations, it is obtainable the originals:

$$
\begin{aligned}
& P_{0}(t)=e^{-\lambda_{1} t} . \\
& P_{1}(t)=\lambda_{1} \frac{e^{k_{1} t}-e^{k_{2} t}}{k_{1}-k_{2}}+\frac{c_{1}}{k_{1} k_{2}}+\left(\frac{c_{1}}{k_{2}\left(k_{2}-k_{1}\right)}\right) e^{k_{2} t}+ \\
& \left(-\frac{c_{1}}{k_{2}\left(k_{2}-k_{1}\right)}-\frac{c_{1}}{k_{1} k_{2}}\right) e^{k_{1} t},
\end{aligned}
$$

where $k_{1}=-\lambda_{1}, k_{2}=-\left(\mu_{1}+\lambda_{2}\right)$, and $c_{1}=\lambda_{1} \mu_{1}$.

The following conditions are imposed:

$$
k_{1} \neq k_{2} \text {. }
$$

The other conditions, when the denominators of the members of the expressions of equation (8) turn to zero, that is when:

$$
k_{2}=0 \text {, }
$$

are impossible; since this root $k_{2}$ is the negative sum of the two initially supposed positive values of the intensities $\lambda_{2}>0$ and $\mu_{1}>0$.

On the contrary, if it had been possible $\lambda_{2}<0$ or $\mu_{1}<0$, although it is mathematically possible, the stated problem would not have had any physical sense.

And on the other hand, if $k_{2}$ had been zero, it would have been another problem setting since in such case

$$
\lambda_{2}=0 \text { and } \mu_{1}=0,
$$

and corresponding changes would have been made starting form Figure 1 and through the procedures of equations (1)-(8).

The same to:

$k_{1}=0, \Rightarrow \lambda_{1}=0$.

Thus,

$$
k_{1} \neq k_{2}, \Rightarrow \mu_{1}+\lambda_{2} \neq \lambda_{1}, k_{1} \neq 0, k_{2} \neq 0 \text {. }
$$

With regard to what happens when:

$$
k_{2} \rightarrow k_{1} \text {. }
$$

In such case equation (8) is a part of the problem with indeterminate forms since the equation's (8) certain members, as fractions, are going to their zeros values both in the numerator and denominators of the corresponding fractions.

In order to make it clear what is happening in the limit:

$$
\lim _{k_{2} \rightarrow k_{1}} P_{1}(t),
$$

there are certain methods applicable, allowing analytical finding the needed result.

According to the limit taking rules:

$$
\begin{aligned}
& \lim _{k_{2} \rightarrow k_{1}} P_{1}(t)=\lim _{k_{2} \rightarrow k_{1}} \lambda_{1} \frac{\left(e^{k_{2} t}-e^{k_{1} t}\right)}{k_{2}-k_{1}}+\lim _{k_{2} \rightarrow k_{1}} \frac{c_{1}}{k_{1} k_{2}}- \\
& \lim _{k_{2} \rightarrow k_{1}} \frac{c_{1} e^{k_{1} t}}{k_{1} k_{2}}-\lim _{k_{2} \rightarrow k_{1}} \frac{c_{1} e^{k_{1} t}}{k_{2}\left(k_{2}-k_{1}\right)}+\lim _{k_{2} \rightarrow k_{1}} \frac{c_{1} e^{k_{2} t}}{k_{2}\left(k_{2}-k_{1}\right)} .
\end{aligned}
$$

Both the second and third members have their definite forms; and for the rest, the well-known in calculus L'Hôpital's (L'Hospital's) rule is applicable, since there are $\frac{0}{0}$ and $\frac{\text { real number }}{0}$ fractions of the kinds of the indeterminate forms.

Therefore:

$$
\begin{gathered}
\lim _{k_{2} \rightarrow k_{1}} P_{1}(t)=\lambda_{1} \lim _{k_{2} \rightarrow k_{1}} \frac{\frac{d}{d k_{2}}\left(e^{k_{2} t}-e^{k_{1} t}\right)}{\frac{d}{d k_{2}}\left(k_{2}-k_{1}\right)}+\frac{c_{1}}{k_{1}^{2}}-\frac{c_{1} e^{k_{1} t}}{k_{1}^{2}}- \\
c_{1} \lim _{k_{2} \rightarrow k_{1}} \frac{\frac{d}{d k_{2}} e^{k_{1} t}}{\frac{d}{d k_{2}} k_{2}\left(k_{2}-k_{1}\right)}+c_{1} \lim _{k_{2} \rightarrow k_{1}} \frac{\frac{d}{d k_{2}} e^{k_{2} t}}{\frac{d}{d k_{2}} k_{2}\left(k_{2}-k_{1}\right)} .
\end{gathered}
$$

Now:

$$
\lim _{k_{2} \rightarrow k_{1}} \frac{\frac{d}{d k_{2}} e^{k_{1} t}}{\frac{d}{d k_{2}} k_{2}\left(k_{2}-k_{1}\right)}=\lim _{k_{2} \rightarrow k_{1}} \frac{0}{2 k_{2}-k_{1}}=0 .
$$


Because the nominator of the above fraction does not depend upon the variated parameter of $k_{2}$ and the denominator is now having some definite value.

Thus:

$$
\begin{aligned}
& \lim _{k_{2} \rightarrow k_{1}} P_{1}(t)=\lambda_{1} \lim _{k_{2} \rightarrow k_{1}} \frac{t e^{k_{2} t}}{1}+\frac{c_{1}}{k_{1}^{2}}-\frac{c_{1} e^{k_{1} t}}{k_{1}^{2}}- \\
& {\left[c_{1} \lim _{k_{2} \rightarrow k_{1}} \frac{\frac{d}{d k_{2}} e^{k_{1} t}}{2 k_{2}-k_{1}}=0\right]+c_{1} \lim _{k_{2} \rightarrow k_{1}} \frac{t e^{k_{2} t}}{2 k_{2}-k_{1}} .}
\end{aligned}
$$

Finally:

$$
\lim _{k_{2} \rightarrow k_{1}} P_{1}(t)=\lambda_{1} t e^{k_{1} t}+\frac{c_{1}}{k_{1}^{2}}-\frac{c_{1} e^{k_{1} t}}{k_{1}^{2}}+c_{1} \frac{t e^{k_{1} t}}{k_{1}}
$$

Analogously to equation (8), the results are obtained for the probability of the state "2":

$$
\begin{aligned}
& P_{2}(t)=\frac{d_{1}}{k_{1} k_{2}}+\left(-\frac{d_{1}}{k_{2}\left(k_{2}-k_{1}\right)}-\frac{d_{1}}{k_{1} k_{2}}\right) e^{k_{1} t}+ \\
& \left(\frac{d_{1}}{k_{2}\left(k_{2}-k_{1}\right)}\right) e^{k_{2} t},
\end{aligned}
$$

where $d_{1}=\lambda_{1} \lambda_{2}$.

The same conditions as for equation (8) are also imposed for equation (9); as well as the same procedure of the indeterminate forms limits takings is applied for Eq. (9). Consideration of such conditions are absent in the reference (Smirnov et al., 1990, Sub-Chapter 15.4, pp. 168-173, especially p. 171 (15.1) and on).

The sought after optimal maintenance periodicity is obtained from the necessary conditions for an extremum existence for the probability of equation (8).

\subsection{Multi-optional effectiveness hybrid functions entropy conditional optimization approach}

Instead of the classical probabilistic approach represented with the procedure of equations (1)-(9) we propose to apply the multi-optional effectiveness hybrid functions entropy conditional optimization doctrine developed hereinafter. Several nuances of the doctrine have been discussed at the previous research publications and are reflected in the corresponding references (Goncharenko, 2017b, 2018a, 2018c). That is a development of subjective analysis (Kasianov, 2013; Goncharenko, 2016, 2017a) for the use of the objective characteristics of an engineering system's functioning.

Consider the following two options for the transportation mean functioning here:

The option \#1 - it is to come optionally into the state " 1 " - the effectiveness function is:

$$
F_{1}=\lambda_{1}-\mu_{1} \text {. }
$$

The option \#2 - it is to come out of (to leave, go out from) the state " 1 " - the effectiveness function is:

$$
F_{2}=\lambda_{2} \text {. }
$$

The optimized objective functional is:

$$
\begin{aligned}
& \Phi_{h}=-\sum_{i=1}^{2} h_{i}\left[F_{i}(\cdot)\right] \ln h_{i}\left[F_{i}(\cdot)\right]+t_{p}^{*} \sum_{i=1}^{2} h_{i}\left[F_{i}(\cdot)\right] F_{i}(\cdot)+ \\
& \gamma\left\{\sum_{i=1}^{2} h_{i}\left[F_{i}(\cdot)\right]-1\right\},
\end{aligned}
$$

where $i$ is number of options, $h_{i}\left[F_{i}(\cdot)\right]$ is hybrid optional function related to the corresponding option effectiveness function (10) or (11), $t_{p}^{*}$ is structural parameter (internal parameter of the process optimality) intrinsic value pertaining to the multi-optional situation with respect to its effectiveness and uncertainty of connected hybrid optional functions, $\gamma$ is structural parameter (weight coefficient or uncertain Lagrange multiplier for the normalizing condition $\sum_{i=1}^{2} h_{i}\left[F_{i}(\cdot)\right]-1$ respectively).

The most important here is to comprehend that there must be some optimality in the framework of the nature things "optionality". The doctrine is similar to seeking after preferences in subjective analysis (Kasianov, 2013; Goncharenko, 2016, 2017a) and applied to hybrid optional optimal distribution findings, likewise in (Goncharenko, 2017b, 2018a, 2018c).

Thus, it is proposed to use an optimization method, which resembles subjective analysis. But the proposed method differs absolutely from subjective analysis (Kasianov, 2013; Goncharenko, 2016, 2017a), since, being applied for the optional effectiveness $F_{i}$, the method does not imply or consider any of active elements of the system at all (Goncharenko, 2017b, 2018a, 2018b, 2018c). Only objectively existing characteristics of the intensities, denoted as $\lambda_{1}, \lambda_{2}$, and $\mu_{1}$ (however, presupposed with the background of the parameter of $t_{p}^{*}$ and of the multioptional effectiveness hybrid functions distribution uncertainty) are utilized.

The first member of the objective functional (12) is the exact distribution uncertainty parameter in the view of the optional hybrid effectiveness functions' entropy, like also discussed at (Goncharenko, 2017b, 2018a, 2018b, 2018c).

Following the proposed here hybrid optional functions entropy optimization doctrine, traced with the contemplation above, the necessary conditions of functional (12) extremum existence is obtained, which yields:

$$
\begin{aligned}
& \frac{\partial \Phi_{h}}{\partial h_{i}}=-\ln h_{i}(\cdot)-1+t_{p}^{*} F_{i}(\cdot)+\gamma=0, \forall i \in \overline{1,2} . \\
& \ln h_{1}(\cdot)-t_{p}^{*} F_{1}(\cdot)=\gamma-1=\ln h_{2}(\cdot)-t_{p}^{*} F_{2}(\cdot) . \\
& \ln h_{1}(\cdot)-t_{p}^{*} F_{1}(\cdot)=\ln h_{2}(\cdot)-t_{p}^{*} F_{2}(\cdot) . \\
& \ln h_{1}(\cdot)-\ln h_{2}(\cdot)=t_{p}^{*}\left[F_{1}(\cdot)-F_{2}(\cdot)\right] . \\
& t_{p}^{*}=\frac{\ln h_{1}(\cdot)-\ln h_{2}(\cdot)}{F_{1}(\cdot)-F_{2}(\cdot)} .
\end{aligned}
$$


In relation (17) the condition of

$$
F_{1}(\cdot) \neq F_{2}(\cdot)
$$

is imposed; similarly to the above considerations it is the equivalent of the conditions for the roots of $k_{1} \neq k_{2}$.

As the effectiveness functions $F_{i}(\cdot)$ are given with Eq. (10) and (11) and the hybrid optional functions $h_{i}(\cdot)$ are deemed to be related with the effectiveness functions:

$$
h_{i}(\cdot)=x F_{i}(\cdot) \text {, }
$$

where $x$ is unknown, uncertain multiplier in type of the Lagrange one, it is found the sought optimal periodicity (solution):

$$
t_{p}^{*}=\frac{\ln \left[\frac{x\left(\lambda_{1}-\mu_{1}\right)}{x\left(\lambda_{2}\right)}\right]}{\left(\lambda_{1}-\mu_{1}\right)-\left(\lambda_{2}\right)}=\frac{\ln \left(\lambda_{1}-\mu_{1}\right)-\ln \left(\lambda_{2}\right)}{\left(\lambda_{1}-\mu_{1}\right)-\left(\lambda_{2}\right)} .
$$

In relation (19) the condition of:

$$
\lambda_{1} \neq \mu_{1}+\lambda_{2} \text {, }
$$

is imposed similarly to the above considerations, since it is equivalent to both $k_{1} \neq k_{2}$ and $F_{1}(\cdot) \neq F_{2}(\cdot)$.

Concerning the case when:

$$
\mu_{1}+\lambda_{2}-\lambda_{1} \rightarrow 0 \text {. }
$$

The limit solution for the optimal maintenance periodicity (19):

$$
\lim _{\lambda_{2} \rightarrow\left(\lambda_{1}-\mu_{1}\right)} t_{p}^{*}=\lim _{\lambda_{2} \rightarrow\left(\lambda_{1}-\mu_{1}\right)}\left[\frac{\ln \left(\frac{\lambda_{2}}{\lambda_{1}-\mu_{1}}\right)}{\lambda_{2}-\left(\lambda_{1}-\mu_{1}\right)}\right]=\frac{0}{0},
$$

corresponding to the case is also having the indeterminate form; therefore it is obtained with the help of the L'Hôpital's (L'Hospital's) rule again.

This yields:

$$
\lim _{\lambda_{2} \rightarrow\left(\lambda_{1}-\mu_{1}\right)} t_{p}^{*}=\lim _{\lambda_{2} \rightarrow\left(\lambda_{1}-\mu_{1}\right)} \frac{\frac{d}{d \lambda_{2}}\left[\mathrm{~h}\left(\frac{\lambda_{2}}{\lambda_{1}-\mu_{1}}\right)\right]}{\frac{d}{d \lambda_{2}}\left[\lambda_{2}-\left(\lambda_{1}-\mu_{1}\right)\right]} .
$$

From where:

$$
\lim _{\lambda_{2} \rightarrow\left(\lambda_{1}-\mu_{1}\right)} t_{p}^{*}=\lim _{\lambda_{2} \rightarrow\left(\lambda_{1}-\mu_{1}\right)} \frac{\frac{d}{d \lambda_{2}}\left[\mathrm{~h} \lambda_{2}-\mathrm{h}\left(\lambda_{1}-\mu_{1}\right)\right]}{1} .
$$

Finally:

$$
\lim _{\lambda_{2} \rightarrow\left(\lambda_{1}-\mu_{1}\right)} t_{p}^{*}=\lim _{\lambda_{2} \rightarrow\left(\lambda_{1}-\mu_{1}\right)} \frac{1}{\lambda_{2}}=\frac{1}{\lambda_{1}-\mu_{1}} .
$$

\section{Results and discussion}

Calculation experiments illustrate the theoretical speculations (1)-(19) of the above sections and subsections of the presented research.

The numerical simulation has been performed for the classical probabilistic method in both the ordinary differential equations system (1) calculation and the system analytical solutions (7), (8), and (9), as well as for the multi-optional doctrine expressed with the Eq. (10)(19). In the modeled case the accepted conditions were $\left.P_{0}\right|_{t=t_{0}}=1,\left.\quad P_{1}\right|_{t=t_{0}}=\left.P_{2}\right|_{t=t_{0}}=0, t_{0}=0$, and other values: $\lambda_{1}=5 \cdot 10^{-3} \mathrm{~h}^{-1} ; \lambda_{2}=1 \cdot 10^{-3} \mathrm{~h}^{-1} ; \mu_{1}=2 \cdot 10^{-3} \mathrm{~h}^{-1}$; $t=0 \ldots 1.5 \cdot 10^{3}$ h. $\mathrm{t}_{\text {opt }} \approx 549.3 \mathrm{~h}$.

The obtained results of the mathematical modeling are shown in Figure 2.

The results depicted in Figure 2 are denoted as follows: a1, a0, a 2 - stand for numerical solutions of the ordinary differential equations system (1), i.e. the probabilities of $\mathrm{P}_{1}(\mathrm{t}), \mathrm{P}_{0}(\mathrm{t}), \mathrm{P}_{2}(\mathrm{t})$ in the corresponding indexing of the possible transportation mean states; $\mathrm{P}_{1}(\mathrm{t}), \mathrm{P}_{0}(\mathrm{t})$, $\mathrm{P}_{00}(\mathrm{t}), \mathrm{P}_{2}(\mathrm{t})$ - analytical solutions for $\mathrm{P}_{1}(\mathrm{t}), \mathrm{P}_{0}(\mathrm{t}), \mathrm{P}_{2}(\mathrm{t})$ by formulae $(7),(8)$, and $(9)$, both $\mathrm{P}_{0}(\mathrm{t})$ and $\mathrm{P}_{00}(\mathrm{t})$ are obtained for probability $\mathrm{P}_{0}(\mathrm{t})$ with the help of characteristic equation and Laplace transformations respectively.

The diagrams illustrating the limits solutions are plotted in Figures 3-5.

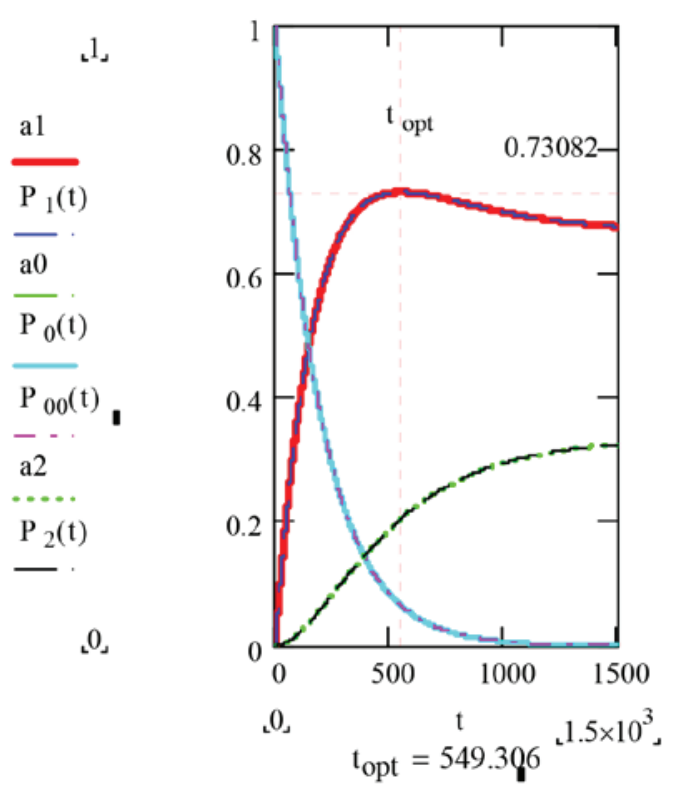

Figure 2. Optimal value of the transportation mean maintenance periodicity

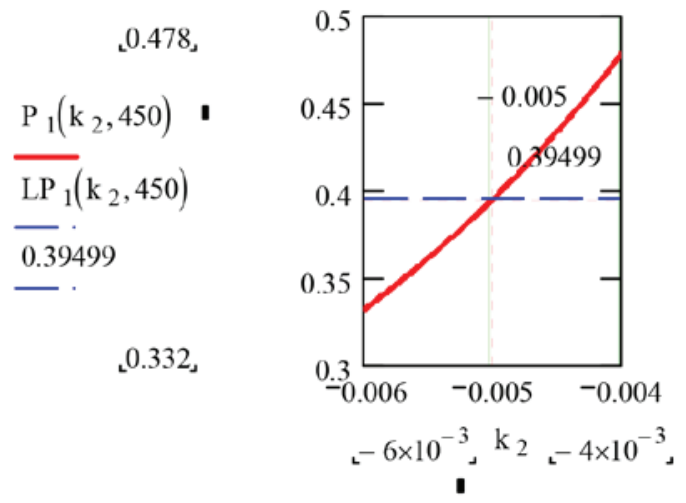

Figure 3. Convergence of the probability limit solution with respect to the roots convergence 
In Figure 3 the probability of $\mathrm{P}_{1}($.$) , Eq. (8), is analyzed$ on the point of its dependence upon the two independent values: the root $k_{2}$ and time $t$. Time is distinguished as $t=450$ for the perception ease.

It can be traced and is easily visible by the $\mathrm{P}_{1}(\cdot)$, Eq. (8), curve that, when $k_{2} \rightarrow\left(k_{1}=-\lambda_{1}=-0.005\right), \mathrm{P}_{1}(\cdot)$, Eq. (8), tendstoitslimit: $\lim _{k_{2} \rightarrow k_{1}} P_{1}(t)=\lambda_{1} t e^{k_{1} t}+\frac{c_{1}}{k_{1}^{2}}-\frac{c_{1} e^{k_{1} t}}{k_{1}^{2}}+c_{1} \frac{t e^{k_{1} t}}{k_{1}}$, denoted as $L P_{1}\left(k_{2}, 450\right)=0.39499$.

Both the right-hand and left-hand limits converge to that value (see the intersection of all the three lines at one point in the middle of the Figure 3 diagram).

The diagrams plotted in Figure 4 are devoted to the same probability $\mathrm{P}_{1}(\cdot)$, Eq. (8), limit convergence but now with respect to time.

It is quite noticeable that in the close neighborhood of the limit: $\lim _{k_{2} \rightarrow k_{1}} P_{1}(t)-\operatorname{LP}_{1}(-0.005, \mathrm{t})$, the probability of $\mathrm{P}_{1}(\cdot)$, Eq. (8), is getting closer and closer to its limiting solution through the whole range of the time $t$ diapason. The curves are practically coinciding, also from bothhands sides.

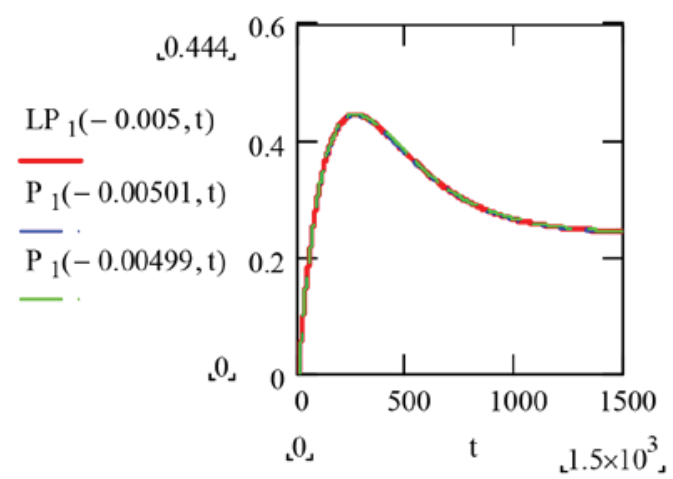

Figure 4. Convergence of the probability limit solution with respect to the roots convergence and through the time interval

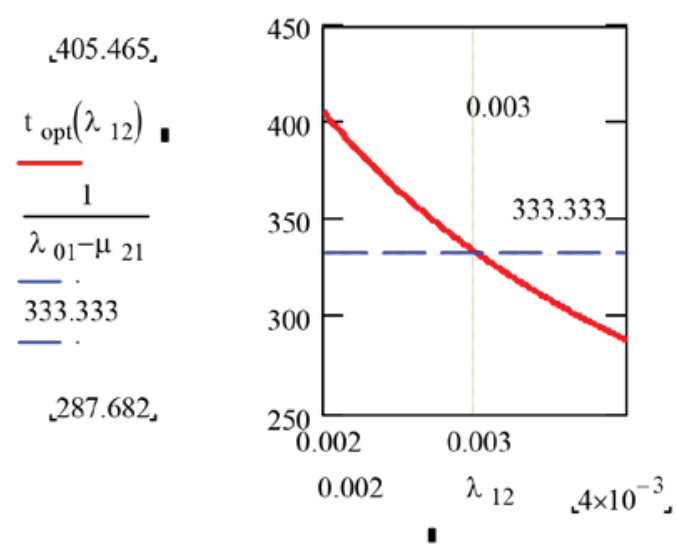

Figure 5. Optimal maintenance periodicity limiting convergence
At last diagrams in Figure 5 illustrate analogical limiting convergence of the optimal maintenance periodicity $t_{p}^{*}$, Eq. (19), designated as $t_{\text {opt }}\left(\lambda_{12}\right), \lambda_{12}$ stands for $\lambda_{2}$ here, when $\mu_{1}+\lambda_{2}-\lambda_{1} \rightarrow 0$, also from both-hand sides. It is seen that when $\lambda_{2} \rightarrow\left(0.003=\lambda_{1}-\mu_{1}\right)$, then $t_{p}^{*}$ tends to its limit value of $\lim _{\lambda_{2} \rightarrow\left(\lambda_{1}-\mu_{1}\right)} t_{p}^{*}=\frac{1}{\lambda_{1}-\mu_{1}}=333.333 . \lambda_{01}$ stands for $\lambda_{1}$ and $\mu_{21}-$ for $\mu_{1}$ in the Figure 5 legend because of the computational designations in the computerized MathCad calculation platform program.

Thus, all results reflected in Figures 3-5 undoubtedly make evidences about the limit solutions existence when the roots have the tendency to converge: $k_{2} \rightarrow k_{1}$. The same statement (the same limits exist from right- and left-hand sides) is appropriate for the situations if $\mu_{1}+\lambda_{2}-\lambda_{1} \rightarrow 0$.

This limits investigation makes one of the differences of the presented research from the theoretical considerations of (Smirnov et al., 1990, Sub-Chapter 15.4, pp. 168173, especially p. 171 (15.1) and on; Goncharenko, 2017b, $2018 \mathrm{~b}, 2018 \mathrm{c}$ ). The other difference is the consideration of the system's possible partial restoration (see Figure 1).

In those cases when the parameters of the general models, similar to portrayed in Figure 1, and described with expressions (1)-(9), have some certain other specific values, the probability of the state "2", Eq. (9), may have the extremum.

In such cases the optimal maintenance periodicities are determined in the framework of the proposed and developed herewith multi-optional conditional optimality doctrine, likewise (10)-(19), too. This is going to be considered in the further sequence of the related investigations.

The developed multi-optional approach (10)-(19) yields the optimal value for the transportation mean optimal maintenance periodicity Eq. (19), which coincides with the traditional method (1)-(9) result obtained as an extremum of dependence (8) (see Figure 2). The maximal value of the probability: $P_{1}(t) \approx 0.7308$.

The important thing here is that the hybrid optional functions uncertainty conditional extremization method uses the hybrid optional functions entropy conditional optimization and is absolutely not the probabilistic approach.

All this instigates searching for a certain scientifically explainable principle for conditions when maintenance of a transportation mean occurs in operational situations which cannot be predicted with the absolute certainty. This is because some damages periodically happen to the transportation mean systems, or an engineering object itself, unexpectedly with the intensities and rates which can be estimated or at lest somehow presupposed in a substantiated manner. 


\section{Conclusions}

From the presented theoretical methods (10)-(19), illustrated with the examples (see Figures $2-5$ ), there is a possibility to conclude that in the studied simplified case (also see Figure 1) it deals with the intrinsic optimal property of the considered process, embodied in the hybrid optional functions uncertainty conditional extremization.

The further research according to the proposed approach has some perspectives for the investigations of conditional optimization and computer simulation in order to illustrate the theoretical speculations expressed with Equations (1)-(19).

\section{References}

Beljatynskij, A., Prentkovskis, O., \& Krivenko, J. (2010). The experimental study of shallow flows of liquid on the airport runways and automobile roads. Transport, 25(4), 394-402. https://doi.org/10.3846/transport.2010.49

Chepizenko, V., Kharchenko, V., \& Pavlova, S. (2013). Synergy of piloted, remotely piloted and unmanned air systems in single air navigation space. Logistics and Transport, 2(18), 77-82. https://doi.org/10.3846/16487788.2015.1057993

Dhillon, B. S. (2006). Maintainability, maintenance, and reliability for engineers. New York: Taylor \& Francis Group. http://doi.org/10.1201/9781420006780

Dmitriyev, S., Koudrin, A., Labunets, A., \& Kindrachuk, M. (2005). Functional coatings application for strengthening and restoration of aviation products. Aviation, 9(4), 39-45.

Fisher, C., \& Hodge, S. (1986). Fisher and Hodge on bunkers. London: Lloyd's of London Press LTD.

Gališanskis, A. (2004). Aspects of quality evaluation in aviation maintenance. Aviation, 8(3), 18-26.

Goncharenko, A. (2016, October 18-20). Several models of artificial intelligence elements for aircraft control. In IEEE 4th International Conference "Methods and Systems of Navigation and Motion Control (MSNMC)" (pp. 224-227). Kyiv, Ukraine.

Goncharenko, A. (2017a). Aircraft operation depending upon the uncertainty of maintenance alternatives. Aviation, 21(4), 126-131. https://doi.org/10.3846/16487788.2017.1415227

Goncharenko, A. (2017b, October 17-19). Optimal UAV maintenance periodicity obtained on the multi-optional basis. In IEEE 4th International Conference "Actual Problems of Unmanned Aerial Vehicles Developments (APUAVD)" (pp. 6568). Kyiv, Ukraine.

Goncharenko, A. (2018a). Optimal controlling path determination with the help of hybrid optional functions distributions.
Radio Electronics, Computer Science, Control, 1(44), 149-158. https://doi.org/10.15588/1607-3274-2018-1-17

Goncharenko, A. (2018b). Aeronautical and aerospace materials and structures damages to failures: Theoretical concepts. International Journal of Aerospace Engineering, 2018, Article ID 4126085, 7 pages. https://doi.org/10.1155/2018/4126085

Goncharenko, A. (2018c, February 20-24). Multi-optional hybrid effectiveness functions optimality doctrine for maintenance purposes. In IEEE 14th International Conference Advanced Trends in Radioelectronics, Telecommunications and Computer Engineering (TCSET-2018) (pp. 771-775). Lviv-Slavske, Ukraine.

Kasianov, V. (2013). Subjective entropy of preferences. Subjective analysis. Warsaw, Poland: Institute of aviation.

Klaas van Dokkum (2005). Ship knowledge covering ship design, construction and operation. Enkhuizen: Dokmar.

Kroes, M. J., Watkins, W. A., Delp, F., \& Sterkenburg, R. (2013). Aircraft maintenance and repair. New York: McGraw-Hill, Education.

Kuiken, K. (2008a). Diesel engines for ship propulsion and power plants from 0 to 100,000 kW (1 part). Onnen: Target Global Energy Training.

Kuiken, K. (2008b). Diesel engines for ship propulsion and power plants from 0 to 100,000 kW (2 part). Onnen: Target Global Energy Training.

Le, H., \& Lappas, I. (2015). Continuing airworthiness: major drivers and challenges in civil and military aviation. Aviation, 19(4), 165-170. https://doi.org/10.3846/16487788.2015.1126909

Nakagawa, T. (2005). Maintenance theory of reliability. London: Springer-Verlag.

Pallos, K. J. (2001). Gas turbine repair technology. Atlanta: GE Energy Services Technology, GE Power Systems.

Smirnov, N. N. et al. (1990). Technical operation of aircraft. Moscow, USSR: Transport.

Smith, D. J. (2005). Reliability, maintainability and risk. Practical methods for engineers. London: Elsevier.

Solomentsev, O., Zaliskyi, M., \& Zuiev, O. (2016). Estimation of quality parameters in the radio flight support operational system. Aviation, 20(3), 123-128. https://doi.org/10.3846/16487788.2016.1227541

Tamarin, Y. A. (2002). Protective coatings for turbine blades. Ohio: ASM International, Materials Park.

Thian, C. V. (2015). Civil and military airworthiness challenges in Asia. Aviation, 19(2), 78-82. https://doi.org/10.3846/16487788.2015.1057993

Wild, T. W., \& Kroes, M. J. (2014). Aircraft powerplants. New York: McGraw-Hill, Education.

Zaporozhets, O., Tokarev, V., \& Attenborough, K. (2011). Aircraft Noise. Assessment, prediction and control. Glyph International, Taylor \& Francis. https://doi.org/10.1201/b12545 